\title{
Single waveguide silicon-organic hybrid modulator
}

\author{
Niklas Hoppe ${ }^{1}$, Christian Rothe ${ }^{2}$, Arda Celik $^{1}$, María Félix Rosa ${ }^{1}$, Wolfgang Vogel ${ }^{1}$, Daniel Widmann ${ }^{1}$, \\ Lotte Rathgeber $^{1}$, M. Carmen Ruiz Delgado ${ }^{3}$, Belén Villacampa ${ }^{4}$, Sabine Ludwigs ${ }^{2}$, and Manfred Berroth ${ }^{1}$ \\ ${ }^{1}$ University of Stuttgart, Institute for Electrical and Optical Communications Engineering, Stuttgart, Germany \\ ${ }^{2}$ University of Stuttgart, Institute for Polymer Chemistry, Stuttgart, Germany \\ ${ }^{3}$ University of Málaga, Department of Physical Chemistry, Málaga, Spain \\ ${ }^{4}$ University of Zaragoza, Department of Condensed Matter Physics, Zaragoza, Spain
}

Correspondence to: Niklas Hoppe (niklas.hoppe@int.uni-stuttgart.de)

Received: 30 December 2016 - Revised: 28 March 2017 - Accepted: 2 May 2017 - Published: 21 September 2017

\begin{abstract}
We present a novel silicon-organic hybrid modulator based on an integrated dual-mode interferometer. The modulator offers a compact, simplified design and enhanced robustness to on-chip fluctuations of temperature compared to conventional Mach-Zehnder based systems. A prototype modulator showing a voltage dependent transmission spectrum is obtained by cladding a dual-mode waveguide in a $250 \mathrm{~nm}$ silicon-on-insulator technology with a customized organic electro-optic layer. Estimated phase shifts and corresponding figures of merit are discussed in this contribution. The used organic layer is based on the guest-host approach with customized donor- $\pi$-acceptor chromophore embedded and poled in a poly(methylmethacrylate) matrix. The presented prototype is to the best of the authors' knowledge the first integrated single waveguide silicon-organic hybrid modulator.
\end{abstract}

\section{Introduction}

In the last decades, the integration and parallelization of electro-optic modulators in combination with higher order modulating schemes led to data rates up to $54 \mathrm{Tbit} \mathrm{s}^{-1}$ transmitted over a single core fiber and a distance of $9150 \mathrm{~km}$ (Cai et al., 2014). One key element is the Mach-Zehnder interferometer modulator (MZM), which is based on electro-optic phase shifters. Different electro-optic effects can be used for the realization of silicon-based phase shifters with electrooptic bandwidths more than $10 \mathrm{GHz}$ (Xiao et al., 2013; Koos et al., 2016). The plasma dispersion effect offers a refractive index change based on the free carrier relocation in silicon. The induced absorption and moderate refractive index change are disadvantages which motivate the integration of electro-optic polymer compounds showing a strong Pockels effect. In the so-called silicon-organic hybrid platform this polymer compound is combined with the silicon-on-insulator (SOI) platform. In-device electro-optic coefficients up to $230 \mathrm{pm} \mathrm{V}^{-1}$ are measured (Koos et al., 2016). In the meantime, MZMs based on electro-optic polymer compounds are working even at elevated temperatures up to $80^{\circ} \mathrm{C}$ demonstrating their stability (Lauermann et al., 2016). Whereas the MZM is an established element based on two waveguide arms, the modulator design can be simplified with the help of a dual-mode or bimodal interferometer (DMI), which is presented e.g. in (Zinoviev et al., 2011; Liu et al., 2014; Hoppe et al., 2017a). Using only one dual-mode waveguide, the novel design is a promising candidate to offer simplified, compact, parallelizable, broadband and thermal stable silicon modulators. Utilizing DMIs in combination with organic layers offers a beneficial thermal stability and robustness to onchip temperature fluctuations for sensor applications (Hoppe et al., 2017a). For that reasons, a prototype DMI modulator is presented in this paper demonstrating the functionality as electro-optic modulator. The paper is structured as follows: The general principle is explained in Sect. 2. The tailor-made organic electro-optic layer is presented in Sect. 3, which is used for the prototype modulator realization as stated out in Sect. 4. Electro-optic measurements are depicted in Sect. 5 where different figures of merit are extracted from the transmission spectra. A conclusion summarizes the achieved results. 


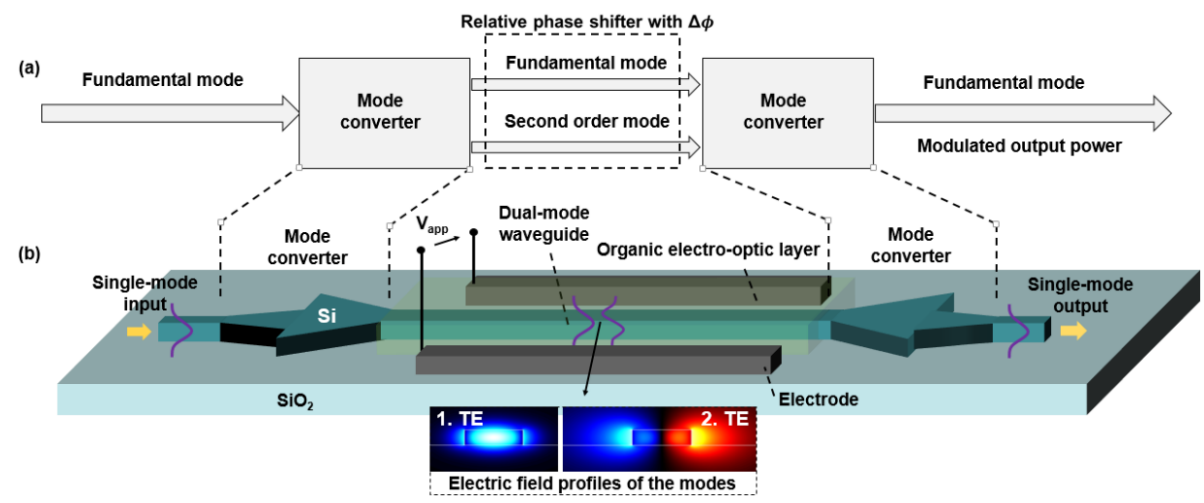

Figure 1. (a) Functional principle of a modulator based on a dual-mode interferometer and (b) a possible schematic structure with two corresponding electric field profiles of the first and second order TE mode in an exemplary dual-mode waveguide. The electric field profiles are calculated by the finite difference method (FDM) mode solver of FIMMWAVE. Here, the two excited mode profiles at the left converter output can look like the shown if the refractive indices of the converter and the dual-mode waveguide claddings are matched.

\section{Device principle}

Instead of using two optical paths that are spatially separated like in a Mach-Zehnder interferometer, a DMI uses two optical paths in a single waveguide by utilizing two different modes. The working principle is based on the fact that two different excited modes in one waveguide are confined differently which results in different penetration depths into the cladding. Therefore, there is different interaction for the two modes with the environment which can be used for modulator applications. Different mode profiles result in different effective refractive indices and finally in a phase difference $\Delta \phi$ between the two modes modulating the output power. Accumulating the phase difference in the same waveguide reveals two main advantages. Firstly, the device size can be decreased in comparison to a MZM that has two separated optical paths. And secondly, the device temperature stability can be increased due to equal environment for both modes. The principle device consists of a dual-mode waveguide between two mode converters (Fig. 1a).

Electrodes are placed in the modulation region for applying an electrical field to an electro-optic organic layer. Twomode excitation is achieved by using mode converters, which can be realized e.g. by laterally shifted tapers (Hoppe et al., 2017a) as depicted in Fig. 1b, by lateral shifted waveguides (Liu et al., 2014), by waveguide steps (Zinoviev et al., 2011), by waveguide gratings (Bruck and Hainberger, 2014) or even simpler by a lateral shift of the external fibers with respect to the center of the grating couplers. Using the last approach, at a special fiber position, the first and second order modes are excited in the following dual-mode waveguide with the same power resulting in a balanced excitation (Hoppe et al., 2015). However, imbalanced excitation can also be beneficial if the two modes suffer from different losses because it can increase the extinction ratio (Hoppe et al., 2017a). Similarly, the two modes are combined at the end of the waveguide by the reverse process using a second mode converter, which includes a single mode output. For modulation, a nonlinear electro-optic layer is deposited on the dual-mode waveguide. The widespread second order mode (see mode profiles in Fig. 1b) with a smaller fill factor predominantly interacts with this layer causing the desired phase difference between the two modes. The effect of the cladding can be described quantitatively by the so-called intrinsic bulk sensitivity in a DMI, given e.g. by Ramirez et al. (2015) as

$\eta_{\text {bulk }}=\frac{\partial\left(\Delta n_{\text {eff }}\right)}{\partial n_{\mathrm{c}}}$

and describes the influence of the cladding refractive index $n_{\mathrm{c}} . \Delta n_{\mathrm{eff}}=n_{\mathrm{eff}, i}-n_{\mathrm{eff}, 1}$ is the difference between the refractive index of the high order mode $(i)$ and the one of the fundamental mode. The phase shift is proportional to the length $L$ of the waveguide where the interaction with the layer takes place. Therefore, Ramirez et al. (2015) defines the bulk sensitivity for the wavelength $\lambda_{0}$ as

$S_{\text {bulk }}=\frac{\partial(\Delta \phi)}{\partial n_{\mathrm{c}}}=\frac{2 \pi L}{\lambda_{0}} \eta_{\text {bulk }}$.

To increase the phase shift, the geometrical length has to be increased by a longer waveguide but it is limited by the maximum device size and losses. The area can be minimized by using a different structure like spiral or meander shaped waveguides (Liu et al., 2014) but losses are still a limitation.

\section{Electro-optic active layer}

The nonlinear optic active layer used in this work is based on the usage of donor- $\pi$-acceptor chromophores which are embedded and poled in a matrix consisting of polymethylmethacrylate (PMMA). This approach is referred to as "guest-host approach" (Dalton et al., 2010; Yesodha et al., 2004). The chromophore consists of a tetrahydroquinoline based unit as donor (Fig. 2a blue) and a tricyanovinylene 
(a)

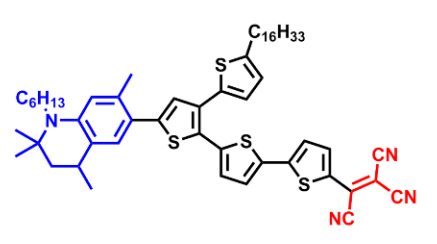

(b)

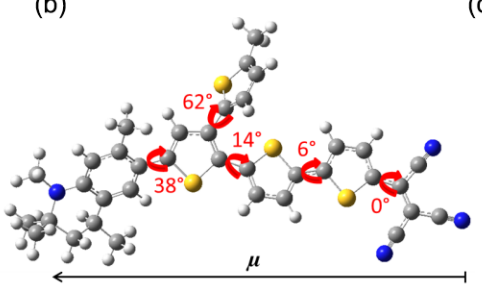

(c)

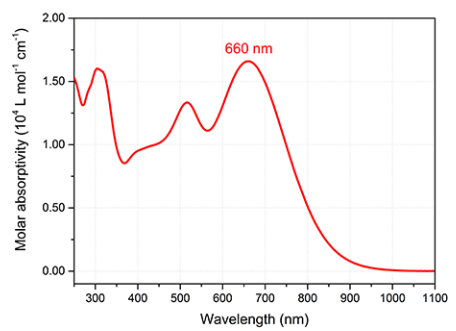

Figure 2. (a) Molecular structure of the nonlinear optical active chromophore with donor (blue), thiophene-linker (black) and acceptor (red). (b) Top view of the optimized ground state molecular structure in dichloromethane solution with the corresponding torsion angles between the building blocks and the dipole moment $\mu$. The simulation was carried out on the M06-2X/6-31G** level, alkyl chains were truncated to methyl groups. (c) Molar absorptivity $\varepsilon$ of the chromophore in dichloromethane solution.

Table 1. Experimental and calculated nonlinear optical properties of the chromophore, with $\mu \beta$ being the product of dipole moment $\mu$ and molecular first hyperpolarizability $\beta, \mu_{\mathrm{g}}$ the ground state dipole moment, $\mu_{\mathrm{e}}$ the excited state dipole moment, $\Delta \mu_{\mathrm{ge}}$ the difference between the ground state and excited state dipole moments, $\mu_{\mathrm{ge}}$ the transition dipole moment and $E_{\max }$ the energy of the transition.

\begin{tabular}{|c|c|c|c|c|c|c|c|}
\hline \multicolumn{2}{|c|}{ Experimental Data } & \multicolumn{6}{|c|}{ Theoretical Data } \\
\hline \multirow[t]{2}{*}{$\mu \beta^{\mathrm{a}}\left(10^{-48} \mathrm{esu}\right)$} & \multirow[t]{2}{*}{$\mu \beta_{0}^{\mathrm{b}}\left(10^{-48} \mathrm{esu}\right)$} & \multirow[t]{2}{*}{$\mu \beta_{0}^{\mathrm{c}}\left(10^{-48} \mathrm{esu}\right)$} & \multicolumn{5}{|c|}{ TD-DFT-calculated ${ }^{\mathrm{d}}$ parameters } \\
\hline & & & $\mu_{\mathrm{g}}(\mathrm{D})$ & $\mu_{\mathrm{e}}(\mathrm{D})$ & $\mu_{\mathrm{ge}}(\mathrm{D})$ & $\Delta \mu_{\mathrm{ge}}(\mathrm{D})$ & $E_{\max }(\mathrm{eV})$ \\
\hline 7700 & 3530 & 6909 & 15.7 & 39.6 & 13.2 & 24.0 & 2.24 \\
\hline
\end{tabular}

${ }^{a}$ Measured by EFISH in dichloromethane at $1.9 \mu \mathrm{m}$ (experimental accuracy $\pm 10 \%$ ).

${ }^{\mathrm{b}}$ Experimental $\mu \beta_{0}$ values calculated using the two level model.

${ }^{c}$ Calculated at the $\mathrm{CPHF}$ level in gas phase//PCM-M06-2X/6-31G**

${ }^{\mathrm{d}}$ Calculated at the M06-2X/6-31G** level in $\mathrm{CH}_{2} \mathrm{Cl}_{2}$.

(TCV) unit as acceptor (Fig. 2a red) which are linked by a $\pi$-conjugated bridge.

Based on our own experience with branched thiophene polymers (Scheuble et al., 2015; Richter et al., 2012) we chose a $\beta$-branched terthiophene (Fig. 2a black) as $\pi$ conjugated building block. The branching on the thiophene linker enhances the temperature stability of the molecule and also acts as a potential hindrance for molecular dipoledipole stacking of the chromophores in the polymer matrix. Density functional theory (DFT) simulations suggest rather planar backbones of the chromophores with the biggest torsion angles being located between the $\beta$-branched thiophene sidechain and the backbone and between the donor and the first $\alpha$-linked thiophene of the terthiophene bridge (see Fig. 2b). The synthesis of the shown chromophore will be published elsewhere. The molecule shows a broad absorption of light between 250 and $950 \mathrm{~nm}$ with a prominent chargetransfer absorption band at a wavelength around $660 \mathrm{~nm}$ (Fig. 2c) resulting in a dark blue color in dichloromethane solution. The second order nonlinear optical properties of the chromophore have been simulated using the CPHF (coupled perturbed Hartree-Fock) approach and also determined experimentally by electric field induced second harmonic generation (EFISH) measurements in dichloromethane solution (see Table 1). The theoretical data are in good accordance with the experimental data and both also lie in the range of known materials with comparable acceptors (Cai et al., 1999; Delgado et al., 2008). Time dependent density functional theory (TD-DFT) calculations reveal that the nonlinear optical (NLO) response seems to arise from the lowest energy allowed absorption. Furthermore, the predicted increase of the dipole moment from 15.7 D in the ground state $\left(\mu_{\mathrm{g}}\right)$ to $39.6 \mathrm{D}$ in the first singlet excited state $\left(\mu_{\mathrm{e}}\right)$ suggests that this transition has a strong charge-transfer nature. Both experimental and theoretical measurements of the new chromophore therefore confirm the potential use in NLO applications.

For the modulators thin films were prepared by dropcasting chloroform solutions $\left(V=20 \mu \mathrm{L}, c=75 \mathrm{mg} \mathrm{mL}^{-1}\right)$ containing PMMA (weight average molecular mass: $\overline{M_{\mathrm{w}}}=\sim$ $350000 \mathrm{~g} \mathrm{~mol}^{-1}$, purchased from Sigma Aldrich) and $24 \mathrm{wt} \%$ of the chromophore on the chips. The films were subsequently dried at room temperature under vacuum. The poling of the chromophores in the films is accomplished directly on the chips.

\section{Prototype realization}

The principle in Hoppe et al. (2015) is used to excite the two lowest order transverse electric (TE) modes in a dualmode waveguide for building a prototype modulator. Hereby, two lateral displaced single mode fibers (SMF 28) above the 

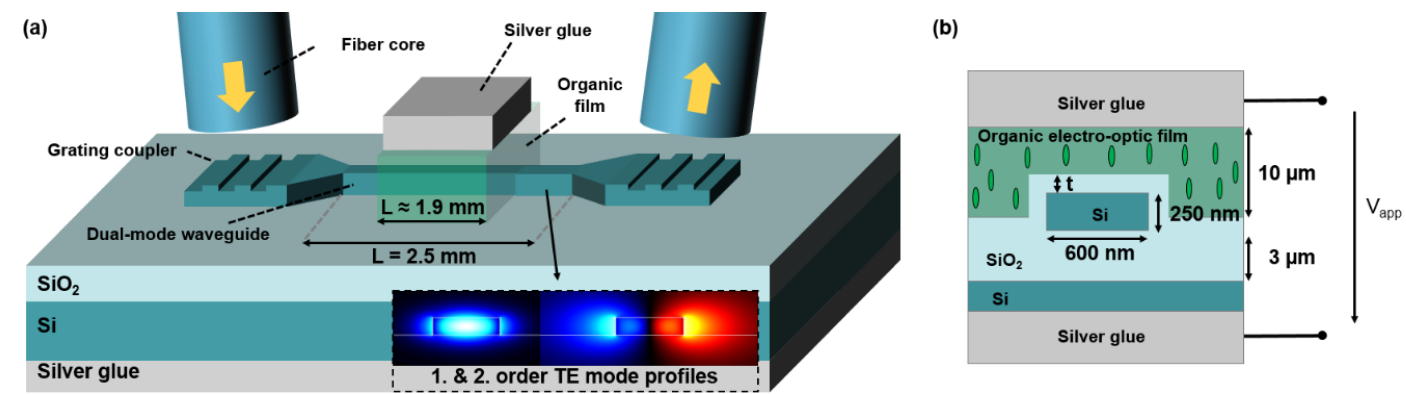

Figure 3. (a) Schematic view of the fabricated modulator prototype. (b) Corresponding cross section of the cladded dual-mode waveguide section in (a) after poling of the organic electro-optic film.

linear input and output grating couplers (see Fig. 3a) act as mode converters as explained in Sect. 2.

Silicon waveguides, tapers and grating couplers are structured by reactive-ion etching steps with optical lithography for the waveguides and electron beam processing for the grating grooves at IMS CHIPS Stuttgart on a Soitec $250 \mathrm{~nm}$ SOI wafer. Etching the original $1 \mu \mathrm{m}$ thick $\mathrm{SiO}_{2}$ passivating layer with the help of a plasma etching step reduces the oxide thickness. Further, the $2500 \mu \mathrm{m}$ long dual-mode waveguide is partly cladded with the electro-optic film presented in Sect. 3 using drop-casting. A silver glue electrode is added on the top of the organic film (see Fig. 3b) to build the top electrode. By adding a second silver glue layer on the backside of the chip, a contact to the weakly doped Si substrate is realized. The two silver glue electrodes enable to orientate the chromophores in the PMMA matrix with the help of a poling procedure. For this procedure, the chip sample is heated up to $120^{\circ} \mathrm{C}$ and a voltage of approximately $400 \mathrm{~V}$ is applied to the electrodes for a period of $1 \mathrm{~h}$.

\section{Characterization of the waveguide channel}

The bimodal waveguide design enables to characterize a geometrical parameter like the $\mathrm{SiO}_{2}$ cladding thickness with high resolution by evaluating the measured transmission spectra. The theoretical background is the free spectral range (FSR) dependence on the group index difference $\Delta n_{\mathrm{g}}$ of the first and second order modes, which can be expressed with the help of

$\Delta n_{\mathrm{g}}=n_{\mathrm{g} 1}-n_{\mathrm{g} 2}$

as

$\mathrm{FSR}=\left|\frac{\lambda_{0}^{2}}{\Delta n_{\mathrm{g}} \cdot L}\right|$,

for wavelengths $\lambda_{0} \gg$ FSR as stated out e.g. in Hoppe et al. (2015). If the length $L$ is known and the FSR is estimated at a center wavelength $\lambda_{0}$ with the help of two minima in the transmission spectrum, the amplitude of $\Delta n_{\mathrm{g}}$ can be calculated. Hereby, $\Delta n_{\mathrm{g}}$ depends for example on the $\mathrm{SiO}_{2}$ cladding thickness and shape. Assuming the shape in Fig. 4a and a width of $550 \mathrm{~nm}$ from a waveguide without electrooptic active cladding, the resulting $\Delta n_{\mathrm{g}}$ is depicted in Fig. $4 \mathrm{~b}$.

Following this procedure, the measured FSR value of $10.92 \mathrm{~nm}$ at $\lambda_{0}=1531 \mathrm{~nm}$ for a waveguide length of $L=$ $2500 \mu \mathrm{m}$ corresponds to a $\mathrm{SiO}_{2}$ thickness of around $180 \mathrm{~nm}$. Note that, if the waveguide is not homogenously cladded, the result is an averaged value. Moreover, for specific geometrical parameters the estimated FSR can correspond to more than one value. This problem can be fixed by evaluating the transmission spectra at different wavelengths and for slightly different waveguide geometries.

Knowing the waveguide geometry and the refractive index of the polymer, the sensitivity to refractive index changes in the electro-optic film can be simulated with the help of the FIMMWAVE software tool. For the waveguide used in the prototype an intrinsic bulk sensitivity value of $7 \%$ is calculated at $\lambda_{0}=1550 \mathrm{~nm}$, whereby the sensitivity of a DMI results from the difference of both mode sensitivities as explained in Sect. 2. The intrinsic bulk sensitivity of the presented prototype can be enhanced to values above $50 \%$ through a better bimodal waveguide design with reduced $\mathrm{SiO}_{2}$ cladding.

\section{Device functionality}

The fabricated device is characterized in an electro-optic measurement setup to demonstrate the modulating functionality. With the help of a tunable laser source (Agilent $81682 \mathrm{~A}$ ), an optical power meter and low frequency probes, the transmission spectrum is measured for different applied voltages $V_{\text {app }}$ between the silver glue electrodes. Hereby, the maximum measured current for the shown voltage range is $0.1 \mu \mathrm{A}$ which implies a good electrical insulation. A voltage dependent shift of the transmission spectrum, which is proportional to the applied voltage, occurs, as depicted in Fig. 5. Changing the polarity of the applied voltage results in an opposite shift in the transmission spectrum. The varying transmission value at a single wavelength demonstrates the modulation of the optical input power. 
(a)

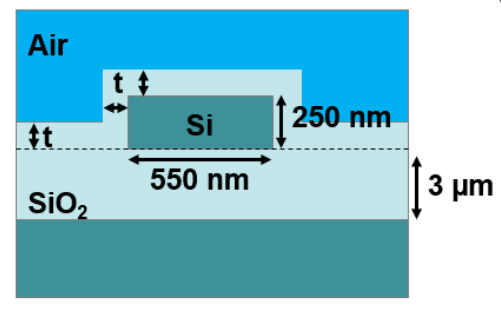

(b)

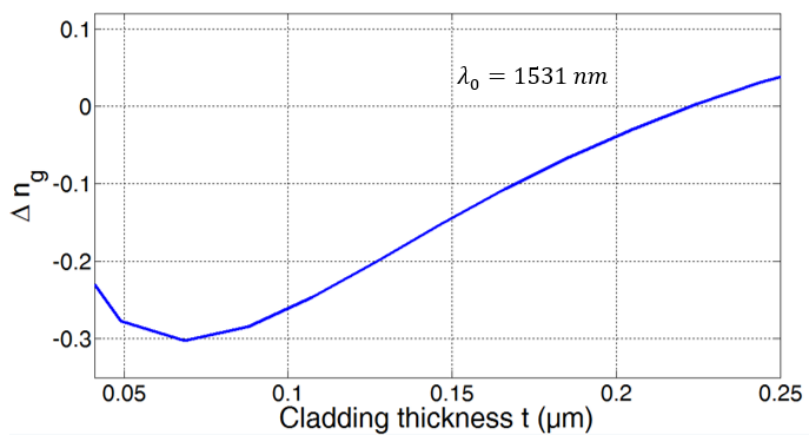

Figure 4. (a) Cross section of a $\mathrm{Si}$ waveguide model with $\mathrm{SiO}_{2}$ cladding. (b) Group index difference for a DMI with the design in (a) versus $\mathrm{SiO}_{2}$ cladding thickness at $\lambda_{0}=1531 \mathrm{~nm}$.
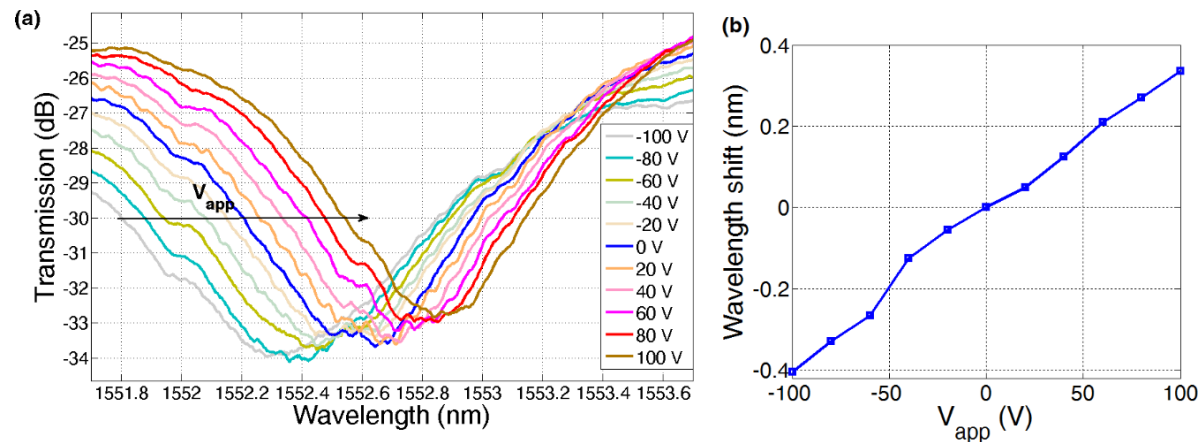

Figure 5. (a) Fiber to fiber transmission spectra of the prototype modulator for different applied voltages $V_{\text {app }}$. (b) Corresponding wavelength shift with FSR being equal to $2.0 \mathrm{~nm}$ at $\lambda_{0} \approx 1552.5 \mathrm{~nm}$.

The resulting phase shift can be calculated following the formula

$\Delta \varphi=\frac{\Delta \lambda}{\mathrm{FSR}} \cdot 2 \pi$,

where, $\Delta \lambda$ refers to the $0 \mathrm{~V}$ spectra and is determined by the local minima in the transmission spectrum. With an achieved $\Delta \varphi$ of $0.2 \pi$ at a voltage of $60 \mathrm{~V}$, the resulting effective electro-optic coefficient $r_{\text {eff }}$ can be estimated, analogous to Koos et al. (2016), as

$r_{\mathrm{eff}}=\frac{d \cdot \lambda_{0} \cdot \Delta \varphi}{V_{\mathrm{app}} \cdot L \cdot n_{\mathrm{EO}}^{3} \cdot \pi} \approx 7.2 \mathrm{pm} \mathrm{V}^{-1}$,

where, the refractive index $n_{\mathrm{EO}}$ of the electro-optic layer is assumed to be 1.7 and the electrode distance $d$ is approximately $13 \mu \mathrm{m}$. Using $r_{33}=3 \cdot r_{13}$ (Teng and Man, 1990) and the intrinsic bulk sensitivity from Sect. 4.1 the on chip $r_{33}$ of the organic layer can be estimated to $300 \mathrm{pm} \mathrm{V}^{-1}$. However, this value should be treated with caution due to possible migration of silver particles into the polymer layer during the poling process and a potentially inhomogeneous oxide cladding shape and thickness on top of the $\mathrm{Si}$ waveguide. The fiber to fiber insertion loss of around $25 \mathrm{~dB}$ is mainly caused by a not optimized grating coupler design and large waveguide losses, which are improved in newer chip generations. E.g. the waveguide loss for the fundamental TE mode in a $400 \mathrm{~nm}$ wide passivated waveguide is reduced from more than 10 to $3.3 \mathrm{~dB} \mathrm{~cm}^{-1}$ in the technology used up to date. The coupling efficiency of the grating couplers can be enhanced by an aperiodic grating design with back side reflector in the used technology to $-0.62 \mathrm{~dB}$ (Sfar Zaoui et al., 2014). To achieve a balanced excitation of the two TE modes, an additional lateral fiber offset with respect to the center of the grating coupler is necessary (Hoppe et al., 2015). This can cause an additional loss, which is dependent on the grating coupler width. E.g. for a $15 \mu \mathrm{m}$ wide $\mathrm{Si}$ grating coupler it is around $3 \mathrm{~dB}$ following the simulations in Hoppe et al. (2015). The extinction ratio of the presented prototype modulator is around $8 \mathrm{~dB}$ (see Fig. 5a).

\section{Conclusions}

We present the novel design of a single waveguide siliconorganic hybrid modulator, which is based on a DMI. The velocities of two optical waveguide modes are differently influenced by changing the refractive index of an organic electrooptic active layer. Adding suitable electrodes results in a voltage dependent transmission, comparable to MZMs. With a fabricated prototype the modulation of the optical power is demonstrated. A customized organic donor- $\pi$-acceptor chromophore is used for obtaining the Pockels effect. The fabri- 
cated sample shows an effective electro-optic coefficient of $7 \mathrm{pm} \mathrm{V}^{-1}$ calculated from the voltage dependent transmission spectrum. The sample is connected by two single mode fibers with the help of grating couplers at the input and output. The fiber to fiber insertion loss of around $25 \mathrm{~dB}$ at a wavelength of $1552 \mathrm{~nm}$ and the extinction ratio of around $8 \mathrm{~dB}$ can be improved by a proper converter design and reduced coupling and waveguide losses.

Data availability. The measurement data and simulation data that support the findings of this study are available in Zenodo with the identifier https://doi.org/10.5281/zenodo.583020 (Hoppe et al., 2017b).

Competing interests. The authors declare that they have no conflict of interest.

Acknowledgements. We would like to thank Philipp Diersing for the measurements of different polymer samples in a modified Teng-Man measurement setup. In addition, we would like to thank IMS CHIPS Stuttgart for fabricating the waveguides used in the prototype. We further thank Klaus Dirnberger for discussions on the design of the chromophores and Thomas Föhn and Wissem Sfar Zaoui for fruitful discussions on the modulator design. The work at the University of Málaga was supported by MINECO (project reference CTQ2015-66897) and Junta de Andalucía (P09FQM-4708). The work at the University of Zaragoza was supported by Spanish Ministry of Science and Innovation, MICINN-FEDER (Project CTQ 2014-52331-R) and the Gobierno de Aragón-Fondo Social Europeo (E39).

Edited by: D. Killat

Reviewed by: R. Kunkel and one anonymous referee

\section{References}

Bruck, R. and Hainberger, R.: Sensitivity and design of grating-assisted bimodal interferometers for integrated optical biosensing, Opt. Express, 22, 32344-32352, https://doi.org/10.1364/OE.22.032344, 2014.

Cai, C., Liakatas, I., Wong, M.-S., Bösch, M., Bosshard, C., Günter, P., Concilio, S., Tirelli, N., and Suter, U. W.: Donor-acceptorsubstituted phenylethenyl bithiophenes: Highly efficient and stable nonlinear optical chromophores, Org. Lett., 1, 1998-2000, https://doi.org/10.1021/o1991118r, 1999.

Cai, J.-X., Sun, Y., Batshon, H. G., Mazurczyk, M., Zhang H., Foursa, D. G., and Pilipetskii, A. N.: 54 Tb/s Transmission over $9,150 \mathrm{~km}$ with Optimized Hybrid RamanEDFA Amplification and Coded Modulation, Optical Communication (ECOC), 2014 European Conference on, 1-3, https://doi.org/10.1109/ECOC.2014.6964261, 2014.

Dalton, L. R., Sullivan, P. A., and Bale, D. H.: Electric Field Poled Organic Electro-optic Materials: State of the Art and Future Prospects, Chem. Rev., 110, 25-55, https://doi.org/10.1021/cr9000429, 2010.

Delgado, M. C. R., Casado, J., Hernández, V., Navarrete, J. T. L., Orduna, J., Villacampa, B., Alicante, R., Raimundo, J.-M., Blanchard, P., and Roncali, J.: Electronic, Optical, and Vibrational Properties of Bridged Dithienylethylene-Based NLO Chromophores, J. Phys. Chem. C, 112, 3109-3120, https://doi.org/10.1021/jp710459c, 2008.

Hoppe, N., Föhn, T., Rosa, M. F., Vogel, W., Zaoui, W. S., Kaschel, M., Butschke, J., Letzkus, F., and Berroth, M.: Integrated dualmode waveguide interferometer, International Conference on Numerical Simulation of Optoelectronic Devices (NUSOD), 155-156, https://doi.org/10.1109/NUSOD.2015.7292869, 2015.

Hoppe, N., Föhn, T., Diersing, P., Scheck, P., Vogel, W., Rosa, M. F., Kaschel, M., Bach, M., and Berroth, M.: Design of an Integrated Dual-Mode Interferometer on $250 \mathrm{~nm}$ Silicon-on-Insulator, IEEE Journal of Selected Topics in Quantum Electronics, 23, 1-8, https://doi.org/10.1109/JSTQE.2016.2618602, 2017 a.

Hoppe, N., Rothe, C., Celik, A., Félix Rosa, M., Vogel, W., Widmann, D., Rathgeber, L., Ruiz Delgado, M. C., Villacampa, B., Ludwigs, S., and Berroth, M.: Data set of a single waveguide silicon-organic hybrid modulator, available at: https://doi.org/10.5281/zenodo.583020, 2017 b.

Koos, C., Leuthold, J., Freude, W., Kohl, M., Dalton, L., Bogaerts, W., Giesecke, A .L., Lauermann, M., Melikyan, A., Koeber, S, Wolf, S., Weimann, C., Muehlbrandt, S., Koehnle, K., Pfeifle, J., Hartmann, W., Kutuvantavida, Y., Ummethala, S., Palmer, R., Korn, D., Alloatti, L., Schindler, P. C., Elder, D. L., Wahlbrink, T., and Bolten, J.: Silicon-Organic Hybrid (SOH) and PlasmonicOrganic Hybrid (POH) Integration, Journal of Lightwave Technology, 34, 256-268, https://doi.org/10.1109/JLT.2015.2499763, 2016.

Lauermann, M., Wolf, S., Hartmann, W., Palmer, R., Kutuvantavida, Y., Zwickel, H., Bielik, A., Altenhain, L., Lutz, J., Schmid, R., Wahlbrink, T., Bolten, J., Giesecke, A. L., Freude, W., and Koos, C.: Generation of 64 GBd 4ASK signals using a siliconorganic hybrid modulator at $80^{\circ} \mathrm{C}$, Opt. Express, 24, 9389-9396, https://doi.org/10.1364/OE.24.009389, 2016.

Liu, Q., Kim, K. W., Gu, Z., Kee, J. S., and Park, M. K.: Singlechannel Mach-Zehnder interferometric biochemical sensor based on two-lateral-mode spiral waveguide, Opt. Express, 22-23, 27910-27920, https://doi.org/10.1364/OE.22.027910, 2014.

Ramirez, J. C., Lechuga, L. M., Gabrielli, L. H., and HernandezFigueroa, H. E.: Study of a low-cost trimodal polymer waveguide for interferometric optical biosensors, Opt. Express, 23, 1198511994, https://doi.org/10.1364/OE.23.011985, 2015.

Richter, T. V., Link, S., Hanselmann, R., and Ludwigs, S.: Design of Soluble Hyperbranched Polythiophenes with Tailor-Made Optoelectronic Properties, Macromol. Rapid Commun., 30, 13231327, https://doi.org/10.1002/marc.200900186, 2009.

Scheuble, M., Gross, Y. M., Trefz, D., Brinkmann, M., Navarrete, J. T. L., Delgado, M. C. R., and Ludwigs, S.: Polythiophenes with Thiophene Side Chain Extensions: Convergent Syntheses and Investigation of Mesoscopic Order, Macromolecules, 48, 70497059, https://doi.org/10.1021/acs.macromol.5b01512, 2015.

Sfar Zaoui, W., Kunze, A., Vogel, W., Berroth, M., Butschke, J., Letzkus, F., and Burghartz, J.: Bridging the gap between optical fibers and silicon photonic integrated circuits, Opt. Express, 22, 1277-1286, https://doi.org/10.1364/OE.22.001277, 2014. 
Teng, C. C. and Man, H. T: Simple reflection technique for measuring the electro-optic coefficient of poled polymers, Appl. Phys. Lett., 56, 1734-1736, https://doi.org/10.1063/1.103107, 1990.

Xiao, X., Xu, H., Li, X., Li, Z., Chu, T., Yu, Y., and Yu, J.: High-speed, low-loss silicon Mach-Zehnder modulators with doping optimization, Opt. Express, 21, 4116-4125, https://doi.org/10.1364/OE.21.004116, 2013.

Yesodha, S. K., Pillai, C. K. S., and Tsutsumi, N.: Stable polymeric materials for nonlinear optics: a review based on azobenzene systems, Prog. Polym. Sci., 29, 45-74, https://doi.org/10.1016/j.progpolymsci.2003.07.002, 2004.
Zinoviev, K. E., González-Guerrero, A., Domínguez, C., and Lechuga, L. M.: Integrated bimodal waveguide interferometric biosensor for label-free analysis, J. Lightwave Technol., 29, 1926-1930, https://doi.org/10.1109/JLT.2011.2150734, 2011. 